PublisherName $\quad$ : BioMed Central

PublisherLocation : London

Publisherl mprintName : BioMed Central

\title{
Biosimulation in Drug Development: Martin Bertau, Erik Mosekilde, Hans V. Westerhoff
}

J ohn Wiley \& Sons, Chichester, UK, 978-3-527-31699-1, 2007, 540, Hardback; £110.00/E154.00

\begin{tabular}{|ll|}
\hline \multicolumn{3}{|l|}{ Articlel nfo } \\
ArticlelD & $: 210$ \\
ArticleDOI & $: 10.1186 / 1479-7364-3-1-98$ \\
ArticleCitationID & $: 98$ \\
ArticleSequenceNumber & $: 1$ \\
ArticleCategory & $:$ Book review \\
ArticleFirstPage & $: 1$ \\
ArticleLastPage & $: 4$ \\
ArticleHistory & $:$ RegistrationDate : 2008-9-1 \\
& $:$ OnlineDate \\
ArticleCopyright & $:$ Henry Stewart Publications2008 \\
ArticleGrants & $:$ \\
ArticleContext & $:$ 402463311 \\
\hline
\end{tabular}

Michael PH Stumpf,Aff1

Corresponding Affiliation: Aff1

Aff1 Division of Molecular Biosciences Centre for Bioinformatics I mperial College London, London, UK

Mathematical modelling has a longstanding place in the biomedical sciences. In particular, mathematical and statistical methods have been instrumental in the development of the disciplines of population genetics and ecology. But over the past 50 or so years there have always been some researchers who have studied biological processes and systems at all levels of organisation, using mathematical and quantitative theoretical methods. The recent rise of the fledgling discipline of systems biology has now, perhaps somewhat belatedly, introduced a wave of mathematical and modelling approaches into the molecular, cellular and physiological sciences. The popularity -- among researchers as well as funding bodies -- of systems biology approaches may well change the biomedical sciences and the training of life- and biomedical scientists profoundly. 
There is a common perception that systems biology research must necessarily involve large teams of researchers, drawn from a variety of complementary backgrounds. In general, this may well be so, particularly in basic and fundamental research. At the applied end, however, it may be possible to conduct proper and leading systems biology research on a much smaller scale. Biosimulation in Drug Development offers several examples where endeavours at the cottage-industry level may provide valuable contributions to the applied side of systems biology. The volume, carefully edited by Martin Bertau, Erik Mosekilde and the indefatigable Hans Westerhoff, has a distinctly theoretical focus, but this is appropriate, as the development of new drugs is one of the areas in modern biomedical research where complications abound, and where mathematical models may be most helpful. The physiological basis of many diseases is only slowly beginning to emerge. Genetic differences among individuals from different ethnic origins, and within populations, give rise to a spectrum of drug response phenotypes. Safe dosages are still predominantly determined in a system of careful trial and error. And to get it wrong can have tragic outcomes for patients and carries huge financial risk for pharmaceutical companies.

The book covers the panoply of modelling applications in drug development. After a brief, and perhaps too generic, introduction to modelling in pharmaceutical systems biology, six chapters cover cellular simulations and the simulation of biological tissues in several medical problems. Generally, these chapters achieve what they set out to do. While they will not enable bench scientists to model their favourite biological system or syndrome, it should be possible to get a flavour of which modelling frameworks are available and appropriate. Occasionally, chapters offer only an overview over a fairly limited range of techniques, and reflect the authors' preferences, rather than the state of the field as a whole. But those already au fait with modelling approaches in biology or the engineering sciences ought to find plenty of stimulation and inspiration, especially in the chapters focusing on particular medical conditions.

The third part of the book contains five chapters in which the predominant focus is on simulating the actions of drugs. Even once the molecular machinery underlying a particular disease has been identified and a suitable intervention has been found in vitro, the in vivo effects, efficacy and safety are hard to predict (or get right). These five chapters focus on simulating the effects of drugs in given tissues and given diseases. They consist mostly of sufficiently detailed case studies, which offer tempting glimpses into this area of research.

Perhaps the most ambitious set of chapters are the four chapters in the last part of the book, which cover applications of biosimulation on a larger scale. A fairly brief chapter which discusses the ins and outs of whole-cell simulation models is followed by a chapter which takes the increasingly important population perspective. There is clear empirical evidence for the hugely varying performance of drug response phenotypes and the existence of genetic polymorphisms that underlie drug metabolism. Appreciating this diversity is key for personalised medicine and it is clear that we are still a long way from combining the molecular, cellular and population levels in biosimulation. Chapters outlining the general use of biosimulation in the drug-development pipeline and in replacing, reducing and refining animal experiments conclude the book.

As far as it is possible to cover an emerging discipline in a single volume, Biosimulation in Drug Development does a good enough job. The book will be of particular interest both to researchers from a mathematical background who are seeking to turn their attention to this area, and to researchers in pharmacogenomics who would like to gain an overview of existing approaches in biosimulation. Compared with the growing literature on modelling biological systems, the discussion of modelling approaches sometimes appears somewhat limited in scope. But, given the complexity of the problem at hand, heuristics (especially if backed up by success) may be preferable to more sophisticated mathematical formalism. As an assessment of the state of a burgeoning field, and as an invitation to join in, this book does an excellent job, but it should also be clear that it offers only a snapshot of a rapidly developing area of research, and the information therein will be outdated very quickly. 